\title{
Therapeutic application of ultrasound: contrast-enhanced thrombolysis in acute ST-elevation myocardial infarction; the Sonolysis study
}

\author{
J. Slikkerveer • G. Veen • Y. Appelman • N. van Royen • \\ O. Kamp
}

Published online: 8 March 2011

(C) The Author(s) 2011. This article is published with open access at Springerlink.com

\begin{abstract}
Contrast enhancement by microbubble infusion has proven its applicability in the field of diagnostic ultrasound. Recent studies also indicate a therapeutic effect of the combined use of ultrasound and microbubbles. Results from animal studies demonstrate that diagnostic ultrasound in combination with intravenous microbubbles can dissolve thrombi. So far, this effect has never been tested in patients with an acute ST-elevation myocardial infarction (STEMI). We recently launched a pilot study in acute STEMI patients to assess safety, feasibility and efficacy of the treatment in this patient group with transthoracic threedimensional diagnostic ultrasound and intravenous microbubbles immediately after prehospital thrombolysis, but prior to primary percutaneous coronary intervention.
\end{abstract}

Keywords Ultrasound $\cdot$ Microbubbles $\cdot$ Myocardial infarction $\cdot$ Facilitated PCI

\section{Background}

The introduction of microbubbles as an ultrasound contrast agent in 1968 increased the diagnostic potential of ultrasound without major drawback [1]. Nowadays, microbubbles are widely accepted as a clinical agent in diagnostic ultrasound. These microbubbles are smaller in size than

\footnotetext{
J. Slikkerveer $(\bowtie)$

Department of Cardiology and Institute of Cardiovascular Research, VU University Medical Center,

Room 4D194, De Boelelaan 1117,

1081 HV Amsterdam, the Netherlands

e-mail: j.slikkerveer@vumc.nl

G. Veen · Y. Appelman • N. van Royen · O. Kamp

Department of Cardiology and Institute of Cardiovascular Research, VU University Medical Center,

Amsterdam, the Netherlands
}

erythrocytes and thus capable of passing through the smallest capillaries. Their acoustic properties make it possible to improve the quality of the local image. Depending on the peak acoustic pressure produced by a source the microbubble scatters in a unique manner. Under influence of low acoustic peak pressures, i.e. mechanical index (MI) below 0.1, the microbubble oscillates linearly, thus enhancing the backscatter. Increasing the peak pressure (MI 0.1-1.0) induces the microbubble to oscillate nonlinearly resulting in harmonic backscatter. Peak pressures with an MI above 1.0 lead to bubble disruption causing transient nonlinear harmonic backscattering. These different types of acoustic behavior can be used for several clinical applications [2].

More recently, it was also shown that combining microbubbles with ultrasound has therapeutic possibilities. Ultrasound alone has proven its therapeutic potential in the field of thromboembolic processes. In vitro and in vivo studies have revealed that the presence of ultrasound increased clot lysis, particularly in low frequency ultrasound with high power [3]. It was implicated that cavitational effects were the main working mechanism behind these results. Cavitation is the creation, vibration and destruction of small bubbles in a medium. Since it was known that microbubbles lower the threshold for acoustic cavitation it was assumed that the addition of microbubbles during ultrasound application could enhance clot lysis even further. Tachibana and Tachibana were the first to prove this principle in vitro [4].

Although cavitation is believed to be the driving force behind this effect, based on the occurrence of microstreaming and shear stress [5], it is also assumed that the generation of reactive oxygen species in endothelial cells [6] and local temperature rise after the microbubble collapse [7] play a pivotal role. However, the full mechanism remains to be resolved [8]. 
After the first positive in vitro results, different in vivo studies have confirmed enhanced clot lysis (Table 1). These studies focused on the macrovascular system and applied different ultrasound frequencies and acoustic pressures. It is known that low frequency ultrasound has a better penetration and thus potentially leads to a better thrombolytic result. Nevertheless, since high frequency ultrasound is already commercially available in the diagnostic field because of its better spatial resolution, the first phase II trial in the neurological field by the CLOTBUST investigators has been performed using high frequency ultrasound. They randomly assigned 126 stroke patients to receive continuous ultrasound or placebo following treatment with intravenous tissue-type plasminogen activator (t-PA) within $3 \mathrm{~h}$ after the onset of symptoms. Their results show a significant increase of complete recanalisation in the ultrasound group compared with placebo [9]. In a spin-off study in stroke patients, microbubbles were added on top of ultrasound and t-PA. This led to a further enhancement of ultrasound augmented thrombolysis, resulting in increased complete recanalisation rates [10].

Currently, the first choice of treatment in patients with acute ST-elevation myocardial infarction (STEMI) is primary percutaneous coronary intervention (PCI) [11]. When PCI is not available, the administration of thrombolytics within $12 \mathrm{~h}$ after onset of symptoms is the optimal approach. Nevertheless, the recanalisation rate after thrombolysis is very low and is also related to more haemorrhagic complications [12].

Until now the combination of diagnostic ultrasound, intravenous microbubbles and thrombolytic therapy has never been tested in STEMI patients admitted for primary PCI. Therefore, we have initiated the first pilot study to determine the safety, feasibility and efficacy of threedimensional (3D) ultrasound and microbubbles to increase the epicardial reperfusion rates in patients with their first acute STEMI presenting in the hospital for primary PCI.

\section{The Sonolysis trial}

We plan to include patients between 18 and 80 years of age diagnosed with their first acute STEMI based on a proximal occlusion of the infarct-related artery. In order to increase the likelihood of a proximal occlusion our inclusion criteria consist of a sum of ST elevation of $6 \mathrm{~mm}$ or more in combined leads and $1 \mathrm{~mm}$ or more ST-elevation in lead V4R in case of an inferior infarction. Patients with clinical instability, previous Q-wave myocardial infarction, contraindication to alteplase, known pulmonary hypertension and known allergy to Luminity ${ }^{\circledR}$ are excluded [13].

Patients are randomised in either a placebo group or a group that receives the ultrasound contrast agent Luminity ${ }^{\mathbb{B}}$.
Randomisation takes place when the ambulance announces a patient with an eligible STEMI. This announcement is done through the unique Lifenet system in the Amsterdam region [14]. A 12-lead ECG of a patient with chest pain is taken by ambulance personnel and sent to a computer in the coronary care unit of the hospital. There, the cardiology resident on call consults the intervention cardiologist and together they check the ECG for inclusion criteria. If the patient is eligible for the study, the ambulance personnel are informed and they check for contraindications to alteplase using a questionnaire. If there are no contraindications patients are pre-treated in the ambulance with a loading dose of aspirin $500 \mathrm{mg}$ iv, heparin $5000 \mathrm{IU}$ iv, and a single bolus alteplase $50 \mathrm{mg}$ iv.

On arrival at the hospital, patients receive, after oral informed consent, either placebo (i.e. $50 \mathrm{ml}$ saline $0.9 \%$ ) without ultrasound, or the ultrasound contrast agent Luminity ${ }^{\circledR}$ during simultaneously pulsatile 3D ultrasound application using the diagnostic ultrasound machine iE33 (Philips, Best, the Netherlands) with a frequency of 1.6 MHz and a MI of 1.18. The matrix 3D probe will be used to obtain 3D full volume images of the aortic root in the parasternal short-axis view to ascertain that the proximal parts of the epicardial coronary artery system are encompassed within the target zone. In order to ensure that the microbubbles replenish around the occlusion of the infarct-related artery, ultrasound is applied intermittently ( $5 \mathrm{~s}$ on, $5 \mathrm{~s}$ off). The microbubbles are infused intravenously for 15 min using a continuous infusion pump at a rate of $200 \mathrm{ml} / \mathrm{h}$.

After $15 \mathrm{~min}$, patients are immediately transported to the catheterisation laboratory where they undergo an emergency angiogram to assess TIMI flow and subsequently primary PCI is carried out when necessary.

Biomarkers and ECG are obtained every $3 \mathrm{~h}$ during the first $24 \mathrm{~h}$. After 2 to 9 days and after 4 months follow-up, a twodimensional echocardiogram and a delayed contrast-enhanced MRI are conducted to measure left ventricular volumes and ejection fraction, wall motion score and scar mass.

Primary endpoint of the study is the TIMI flow grade to document patency of the culprit vessel.

Safety is assessed by documenting adverse events and serious (cardiac) adverse events during treatment and followup of 4 months. Death, cardiogenic shock, ventricular fibrillation, sustained ventricular tachycardia, major bleeding (e.g. when packed cells are needed) and recurrent myocardial infarction are marked as serious cardiac adverse events.

Feasibility is based on the successful accomplishment of the treatment protocol prior to PCI.

Fig. 1 shows a case example of a patient who received active therapy with diagnostic 3D ultrasound and intravenous microbubbles. An acute STEMI of the anterior wall is visible on the ECG obtained in the ambulance. The sum of 


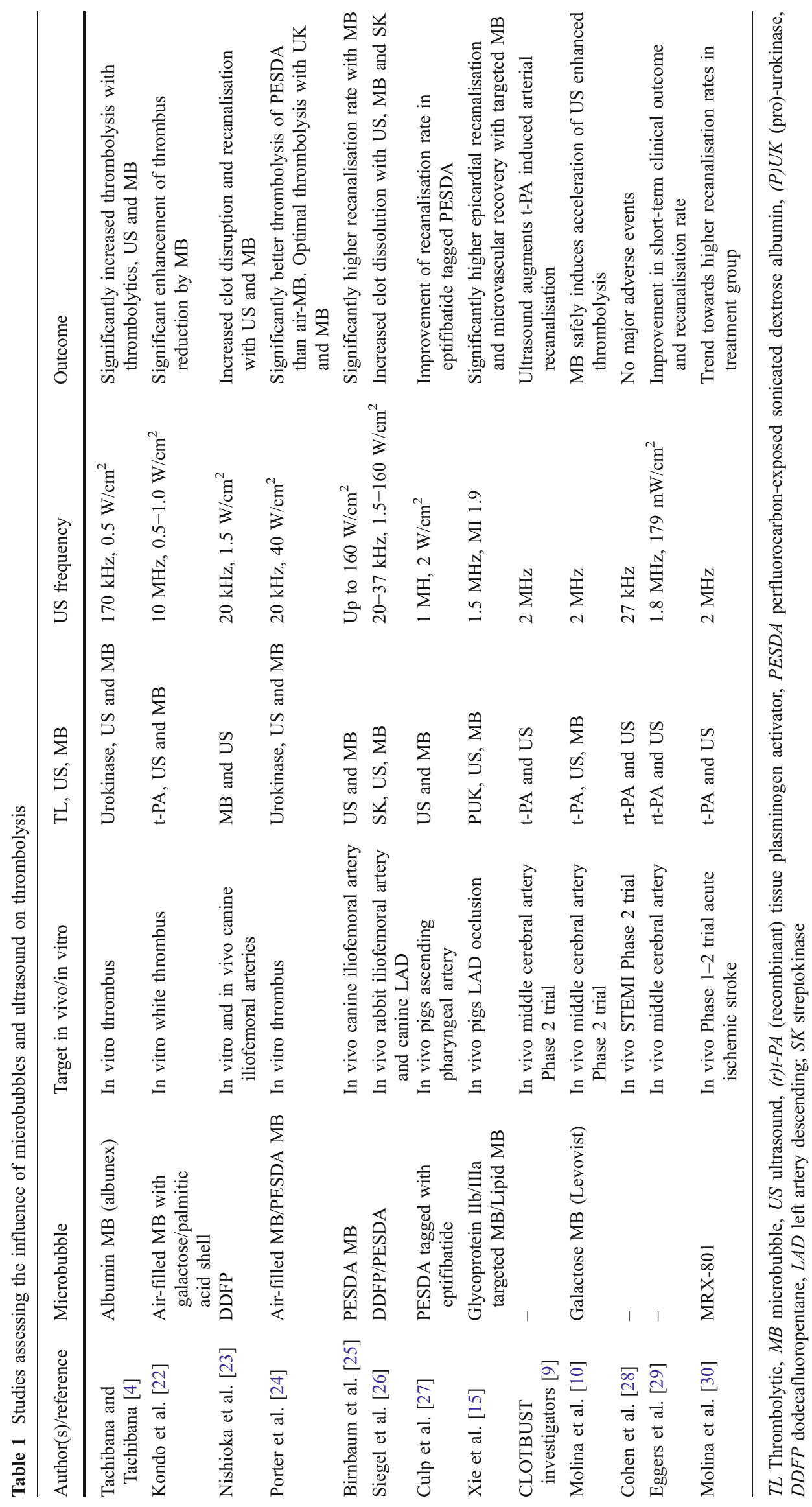




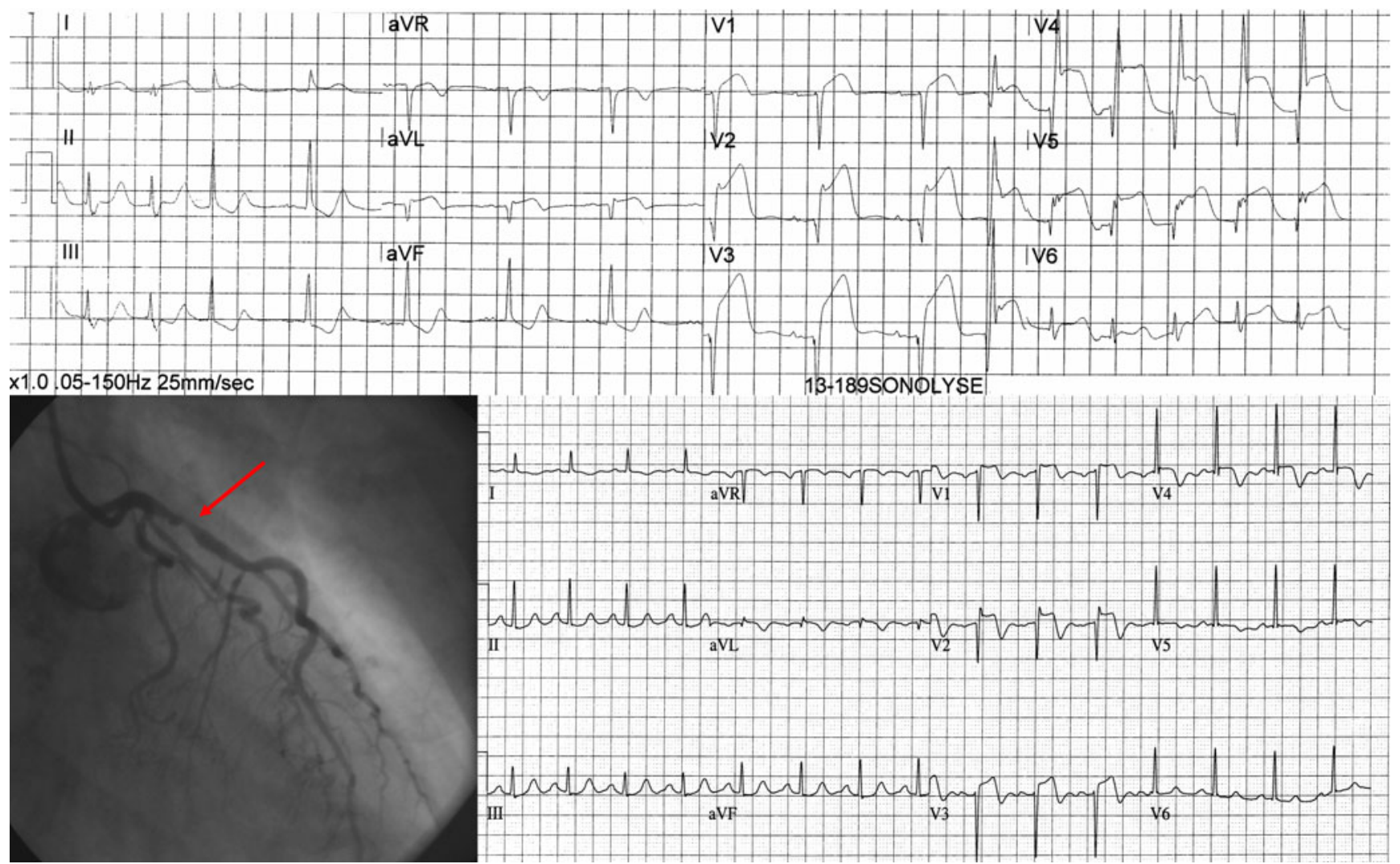

Fig. 1 Example of a patient. The top ECG shows an acute anterior wall infarction. The patient received active treatment and subsequently underwent angiography which revealed a TIMI III flow with more

ST elevation is $28 \mathrm{~mm}$ which meets the inclusion criteria. At the end of ultrasound treatment the ECG showed an accelerated idioventricular rhythm (not shown) and the patient noticed chest pain relief. On the emergency angiogram TIMI III flow of the left descending artery was seen, prior to PCI. Since there was still a more than $70 \%$ stenosis, PCI was performed. ECG at 60 min after primary PCI shows more than $70 \%$ ST resolution and maximum CK-MB was $104 \mu \mathrm{g} / \mathrm{l}$. No adverse events occurred during treatment and at 4 months follow-up.

\section{Future application}

\section{No-reflow}

The aim of this pilot study is to assess safety, feasibility and efficacy of the treatment with diagnostic 3D ultrasound and intravenous microbubbles in the setting of an acute STEMI with pre-treatment of low-dose thrombolytics in the ambulance, focusing on the epicardial reperfusion rate under influence of ultrasound and microbubbles compared with placebo.

Interestingly, until recently, research was focused on recanalisation of the thrombotic occluded epicardial artery. than $70 \%$ occlusion of the proximal LAD. A stent was placed and the ECG 60 min after primary PCI showed more than $70 \%$ ST resolution

In an in vivo pig study it was observed that the microvascular flow improved after treatment of the risk area despite absence of epicardial reperfusion [15]. Preliminary data in a second pig experiment by the same group using 3D ultrasound confirmed these results [16]. Not only does the combination of ultrasound and microbubbles have an effect on the epicardial coronary artery system, it might also have a positive effect on microvascular flow after acute STEMI and thus serve as an adjuvant therapy in patients with noreflow. A possible explanation might be the local release of nitric oxide under influence of ultrasound, resulting in an increased capillary diameter and thus improvement of local perfusion [17]. Furthermore, the earlier mentioned temperature rise and creation of microjets under influence of cavitation might also influence the pathogenetic mechanism of no-reflow in the microvasculature [18].

Drug and gene delivery

On top of the enhanced thrombolytic effect, the combination of ultrasound and microbubbles has also proven its potential in the field of drug and gene delivery. The local destruction of microbubbles under the influence of ultrasound results in the enhanced uptake of dextran molecules. 
It was shown by Meijering et al. that pore formation as well as endocytosis are the two main mechanisms of uptake [19]. This implicates possible pathways for local drug and gene delivery. Especially, in combination with the option to target the microbubbles to increase the local concentration in the area of interest, this might enhance the potential effect of stem cell therapy in the setting of an acute MI [20]. In a mouse MI model, Fujii and colleagues have managed a successful local delivery of vascular endothelial growth factor (VEGF) and stem cell factor (SCF) incubated in microbubbles using ultrasound for local destruction, resulting in improved myocardial perfusion and ventricular function [21].

\section{Conclusion}

This first Sonolysis study might stimulate future research and technical innovations to improve the treatment of STEMI patients to obtain more patency at an earlier stage in the ambulance. Furthermore, the combination of ultrasound and intravenous microbubbles might also be useful in the setting of primary PCI for treatment of no-reflow.

Thus, the application of ultrasound and microbubbles in the field of cardiology are very widespread and hold great opportunities for future therapeutic interventions.

Acknowledgements This study is supported by a grant of the KNAW (Royal Netherlands Academy of Arts and Sciences).

Interuniversity Cardiology Institute of the Netherlands, ICIN project 49.02

Open Access This article is distributed under the terms of the Creative Commons Attribution Noncommercial License which permits any noncommercial use, distribution, and reproduction in any medium, provided the original author(s) and source are credited.

\section{References}

1. Gramiak R, Shah PM. Echocardiography of the aortic root. Invest Radiol. 1968;3(5):356-66.

2. Becher H, Burns PN. Contrast Agents for Echocardiography: Principles and Instrumentation. Handbook of Contrast Echocardiography: Left Ventricular Function and Myocardial Perfusion. Frankfurt and New York: 2000. p. 1-45.

3. Siegel RJ, Fishbein MC, Forrester J, et al. Ultrasonic plaque ablation. A new method for recanalization of partially or totally occluded arteries. Circulation. 1988;78(6):1443-8.

4. Tachibana K, Tachibana S. Albumin microbubble echo-contrast material as an enhancer for ultrasound accelerated thrombolysis. Circulation. 1995;92(5):1148-50.

5. van Wamel A, Bouakaz A, Versluis M, et al. Micromanipulation of endothelial cells: ultrasound-microbubble-cell interaction. Ultrasound Med Biol. 2004;30(9):1255-8.

6. Juffermans LJ, Dijkmans PA, Musters RJ, et al. Transient permeabilization of cell membranes by ultrasound-exposed microbubbles is related to formation of hydrogen peroxide. Am J Physiol Heart Circ Physiol. 2006;291(4):H1595-601.
7. Wu J. Temperature rise generated by ultrasound in the presence of contrast agent. Ultrasound Med Biol. 1998;24(2):267-74.

8. Juffermans LJM. Ultrasound and microbubble-targeted delivery of drugs and genes VU University Medical Center, Amsterdam, the Netherlands; 6-4-2009.Ph.D. dissertation

9. Alexandrov AV, Molina CA, Grotta JC, et al. Ultrasoundenhanced systemic thrombolysis for acute ischemic stroke. N Engl J Med. 2004;351(21):2170-8.

10. Molina CA, Ribo M, Rubiera M, et al. Microbubble administration accelerates clot lysis during continuous 2-MHz ultrasound monitoring in stroke patients treated with intravenous tissue plasminogen activator. Stroke. 2006;37(2):425-9.

11. Keeley EC, Boura JA, Grines CL. Primary angioplasty versus intravenous thrombolytic therapy for acute myocardial infarction: a quantitative review of 23 randomised trials. Lancet. 2003;361 (9351):13-20.

12. An international randomized trial comparing four thrombolytic strategies for acute myocardial infarction. The GUSTO investigators. N Engl J Med. 1993;329(10):673-82.

13. Slikkerveer J, Dijkmans PA, Sieswerda GT, et al. Ultrasound enhanced prehospital thrombolysis using microbubbles infusion in patients with acute ST elevation myocardial infarction: rationale and design of the Sonolysis study. Trials. 2008;9:72.

14. Adams R, Appelman Y, Bronzwaer JG, et al. Implementation of a prehospital triage system for patients with chest pain and logistics for primary percutaneous coronary intervention in the region of Amsterdam, the Netherlands. Am J Cardiol. 2010;106 (7):931-5.

15. Xie F, Lof J, Matsunaga T, et al. Diagnostic ultrasound combined with glycoprotein IIb/IIIa-targeted microbubbles improves microvascular recovery after acute coronary thrombotic occlusions. Circulation. 2009;119(10):1378-85.

16. Slikkerveer J, Xie F, Kamp O, et al. Improvement in myocardial blood volume and ultimate infarct size despite persistent epicardial occlusion using three-dimensional transthoracic ultrasound and intravenous non-targeted microbubbles. Eur Heart J. 2010;31 suppl 1:873-1071.

17. Siegel RJ, Suchkova VN, Miyamoto T, et al. Ultrasound energy improves myocardial perfusion in the presence of coronary occlusion. J Am Coll Cardiol. 2004;44(7):1454-8.

18. Niccoli G, Burzotta F, Galiuto L, et al. Myocardial no-reflow in humans. J Am Coll Cardiol. 2009;54(4):281-92.

19. Meijering BD, Juffermans LJ, van Wamel A, et al. Ultrasound and microbubble-targeted delivery of macromolecules is regulated by induction of endocytosis and pore formation. Circ Res. 2009;104 (5):679-87.

20. Juffermans LJ, Meijering DB, van Wamel A, et al. Ultrasound and microbubble-targeted delivery of therapeutic compounds: ICIN Report Project 49: Drug and gene delivery through ultrasound and microbubbles. Neth Heart J. 2009;17(2):82-6.

21. Fujii H, Sun Z, Li SH, et al. Ultrasound-targeted gene delivery induces angiogenesis after a myocardial infarction in mice. JACC Cardiovasc Imaging. 2009;2(7):869-79.

22. Kondo I, Mizushige K, Ueda T, et al. Histological observations and the process of ultrasound contrast agent enhancement of tissue plasminogen activator thrombolysis with ultrasound exposure. Jpn Circ J. 1999;63(6):478-84.

23. Nishioka T, Luo H, Fishbein MC, et al. Dissolution of thrombotic arterial occlusion by high intensity, low frequency ultrasound and dodecafluoropentane emulsion: an in vitro and in vivo study. $\mathrm{J}$ Am Coll Cardiol. 1997;30(2):561-8.

24. Porter TR, LeVeen RF, Fox R, et al. Thrombolytic enhancement with perfluorocarbon-exposed sonicated dextrose albumin microbubbles. Am Heart J. 1996;132(5):964-8.

25. Birnbaum Y, Luo $H$, Nagai $T$, et al. Noninvasive in vivo clot dissolution without a thrombolytic drug: recanalization of throm- 
bosed iliofemoral arteries by transcutaneous ultrasound combined with intravenous infusion of microbubbles. Circulation. 1998;97 (2): $130-4$.

26. Siegel RJ, Atar S, Fishbein MC, et al. Noninvasive transcutaneous low frequency ultrasound enhances thrombolysis in peripheral and coronary arteries. Echocardiography. 2001;18 (3): $247-57$

27. Culp WC, Porter TR, Lowery J, et al. Intracranial clot lysis with intravenous microbubbles and transcranial ultrasound in swine. Stroke. 2004;35(10):2407-11.
28. Cohen MG, Tuero E, Bluguermann J, et al. Transcutaneous ultrasound-facilitated coronary thrombolysis during acute myocardial infarction. Am J Cardiol. 2003;92(4):454-7.

29. Eggers J, Konig IR, Koch B, et al. Sonothrombolysis with transcranial color-coded sonography and recombinant tissue-type plasminogen activator in acute middle cerebral artery main stem occlusion: results from a randomized study. Stroke. 2008;39(5):1470-5.

30. Molina CA, Barreto AD, Tsivgoulis G, et al. Transcranial ultrasound in clinical sonothrombolysis (TUCSON) trial. Ann Neurol. 2009;66(1):28-38. 